\title{
Computer Modeling of Carbon Metabolism Enables Biofuel Engineering
}

In an effort to reduce the cost of biofuels, the National Renewable Energy Laboratory (NREL) has merged biochemistry with modern computing and mathematics. The result is a model of carbon metabolism that will help researchers understand and engineer the process of photosynthesis for optimal biofuel production.

Organisms like green algae, grasses, and trees use photosynthesis to transform light energy and carbon dioxide into chemicals_chemicals that can be turned back into energy when used as biofuels or feedstocks for biofuel production. Researchers at NREL have set out to make photosynthesis more efficient, so that more energy can be captured as biofuels.

To improve the efficiency of photosynthetic energy capture, the researchers needed a working model of the organisms' key metabolism. Specifically, they needed a detailed physical and chemical model of how carbon and energy flow through an organism's processes. This model would make it possible to understand the complex interactions that occur within cells that perform photosynthesis. NREL aimed to build this model in a way that would make it easy to share with other scientists and permit high-performance computer simulations.

NREL scientists also wanted the capability to accurately predict the optimal outcomes of metabolic engineering in the laboratory. If hypotheses could be tested in computer simulations, then the scientists could more accurately predict the outcomes of biofuel engineering experiments.

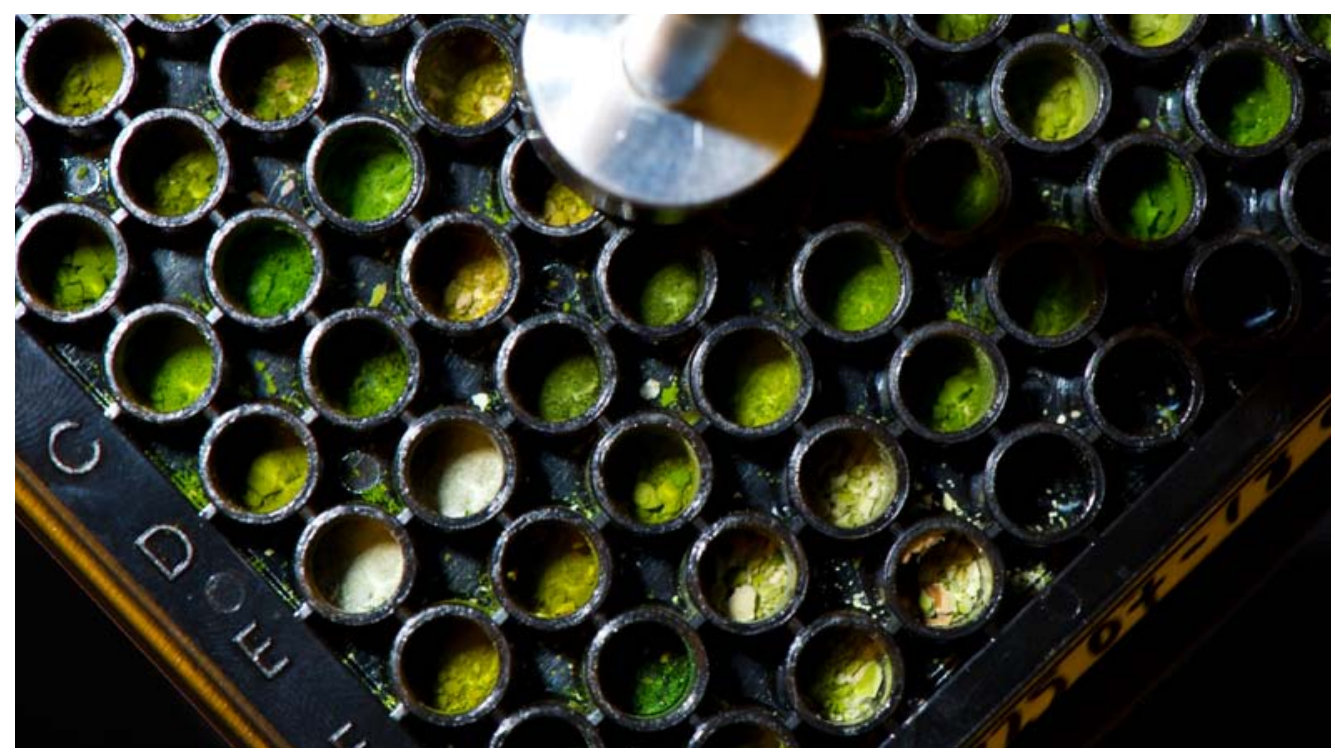

These test tubes in an NREL algae lab hold extractions from several algal strains. NREL is studying carbon metabolism in organisms such as algae to learn how to optimize these organisms to serve as feedstocks for biofuel production. Photo by Dennis Schroeder, NREL/PIX 18228

The Spectrum of

Clean Energy Innovation

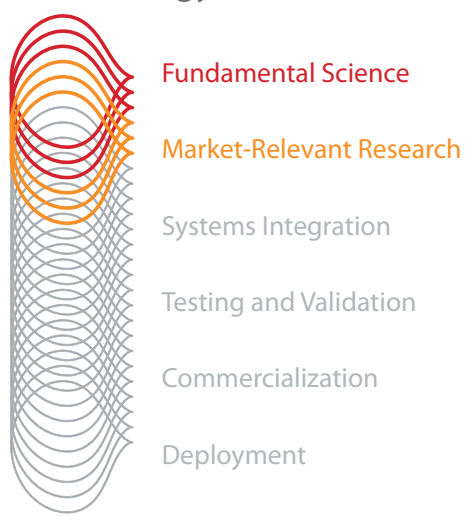

Through deep technical expertise and an unmatched breadth of capabilities, NREL leads an integrated approach across the spectrum of renewable energy innovation. From scientific discovery to accelerating market deployment, NREL works in partnership with private industry to drive the transformation of our nation's energy systems.

This case study illustrates NREL's innovations in Fundamental Science through Market-Relevant Research.

\section{INREL \\ NATIONAL RENEWABLE ENERGY LABORATORY}

NREL is a national laboratory of the U.S. Department of Energy, Office of Energy Efficiency and Renewable Energy, operated by the Alliance for Sustainable Energy, LLC. 


\section{Powerful Tools for a Big Job}

The U.S. Department of Energy (DOE) supported this effort through the Scientific Discovery through Advanced Computing (SciDAC) Program. With that backing, an NREL research and supercomputing team set out to assemble a model of carbon metabolism that is common to most photosynthetic organisms.

The process of metabolism includes a series of chemical steps that use catalysts to speed up chemical reactions. Most catalysts used in metabolism are enzymes, which are cellular proteins. The rates of these enzymatic reactions vary with the concentrations of cellular chemicals (metabolites), and other chemicals that regulate the reaction (regulators).

These rates can be described mathematically with ordinary differential equations (ODEs). When scientists created a model of all the metabolic reactions that perform photosynthesis in a cell, the result was a set of interacting ODEs. The task required moving beyond models that simulated individual reactions to a larger model that simulates dynamic, biochemical networks in cells. That leap in scope is what necessitated the use of high-performance computers.

The NREL team started by constructing a model that included the six common chemical processes that many organisms use to change carbon into energy. Those processes are glycolysis, the tricarboxylic acid cycle, the reductive pentose phosphate pathway, oxidative phosphorylation, starch degradation, and fermentation reactions. It was important that the model accurately reflect the chemistry and physics of carbon metabolism, so it was vital to incorporate the full complexity of the enzymatic rate equations described in the scientific literature.

The model was also formulated so that chemical interactions could be grouped according to their locations within the cell. The researchers used Chlamydomonas reinhardtii, an extensively studied form of green alga, as a model cell.

\section{A Super-Sized Computer Model}

When finished, the NREL team had one of the largest models ever formulated at this level of detail. It includes 149 compounds, 65 enzymes, 688 parameters, and 114 reactions. The model incorporates metabolic transformations in detail, accounting for factors such as thermodynamics, or the amount of energy exchanged in each reaction; kinetics, or the change in reaction rate with changes in concentrations; the concentration of the catalysts; and regulation of the reactions by enzymes.

NREL researchers created the model with a standardized biochemical expression language so it could be easily maintained and shared with the scientific community. They also wrote software to simulate the model with the High Performance Systems Biology Toolkit (HiPer SBTK). This toolkit was developed at NREL, as part of the project, to enable parallel simulations on high-performance computing machines used by many researchers.

The model involves many physical parameters that scientists must know to manipulate an organism's metabolism for optimizing biofuel production. Scientists must also understand how the system responds to changes in these parameters. Large-scale simulations and optimizations with HiPer SBTK on the Red Mesa supercomputer-a collaboration between NREL and Sandia National Laboratories, located at Sandia—will allow scientists to better understand how to manipulate the carbon-to-energy process in biochemical systems. With such knowledge, the biochemist in the laboratory can change, add, or remove genes that direct the behavior of enzymatic catalysts, creating desirable metabolic behaviors for biofuel production. Furthermore, this systems biochemistry approach will enable greater fundamental understanding of biochemical control and dynamics. Such understanding can be used for other types of metabolic engineering.

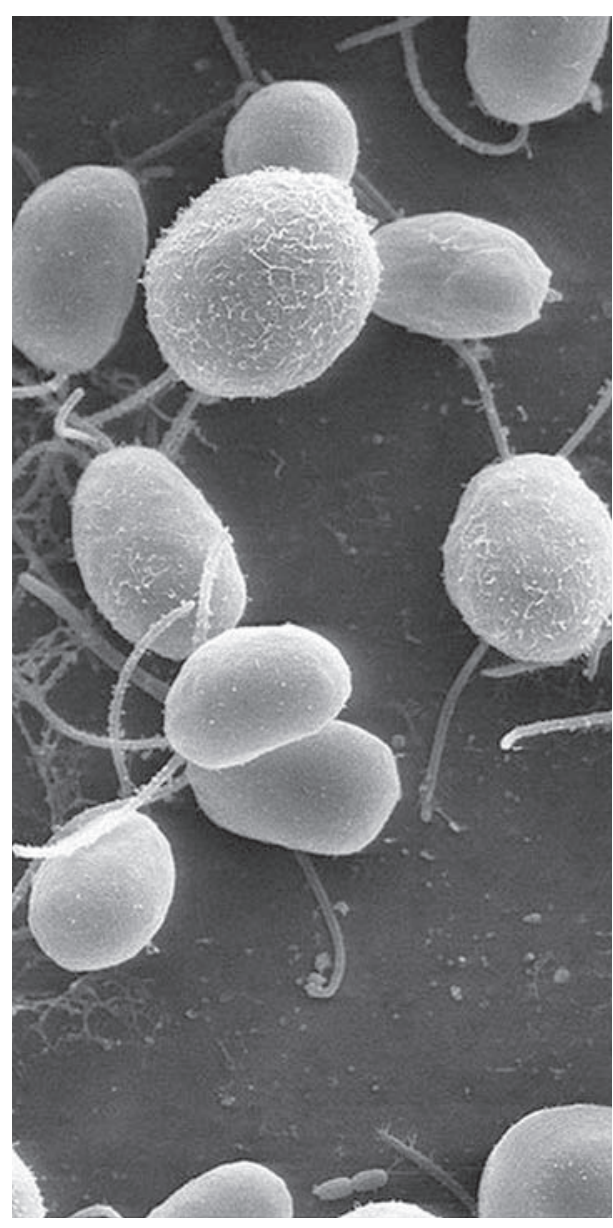

One of the components that NREL scientists needed to create a model of carbon metabolism was a model cell. They chose the cell of Chlamydomonas reinhardtii, a green alga studied extensively at NREL.

Image from Dartmouth Electron Microscope Facility, Dartmouth College, NREL/PIX 19459

\section{National Renewable Energy Laboratory}

1617 Cole Boulevard

Golden, Colorado 80401

303-275-3000 • www.nrel.gov

NREL is a national laboratory of the U.S. Department of Energy, Office of Energy Efficiency and Renewable Energy, operated by the Alliance for Sustainable Energy, LLC.

NREL/FS-6A42-51558 • September 2011

Printed with a renewable-source ink on paper containing at least $50 \%$ wastepaper, including $10 \%$ post consumer waste.

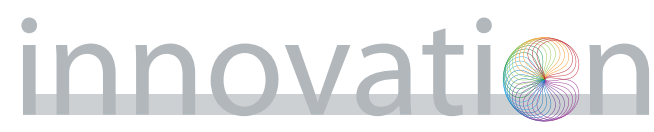

